\title{
Trans people's reproductive options and outcomes
}

\section{Christina Richards, ${ }^{1}$ Leighton Seal ${ }^{2}$}

\section{INTRODUCTION}

Trans people are those people whose sex assigned at birth does not align with their gender identity - a condition that can cause marked distress. Consequently, many trans people seek to change their gender, often permanently. Most usually that change is to male or female although sometimes the change is to a non-binary gender form. However, as the last of these is less usual this commentary will consider only trans people who identify as male or female.

The options available to trans people vary according to cultural context and so this commentary considers such matters from a context in which hormonal and surgical assistance is comparatively readily available. Within such contexts, people who choose to transition often use hormones and surgery to create a body that is more congruent with their perception of themselves as men or women.

Thus people assigned as female at birth who identify and live as men (trans men) may take testosterone in order to grow facial hair and thicker body hair, increase musculature, create a deeper voice and ensure the cessation of menses. If they have the genetic propensity they will go bald. They will commonly have surgery to remove their breasts and produce a male chest contour and, less commonly, to have a surgically constructed penis. They will also be likely to have a hysterectomy and oophorectomy.

Those people assigned as male at birth who identify and live as women (trans women) may have androgen suppression as well as estrogens in order to develop breasts and a more female body contour. Body hair may lessen, but facial hair will need removing by electrolysis. Scalp hair loss will stop, but hair will not regrow and hairpieces may therefore be used. The masculine voice is not affected by estrogens and speech therapy and sometimes surgery can be used to address this. Approximately $60 \%$ of trans women request augmentation mammoplasty, ${ }^{1}$ in part because the phenotypic male chest is larger than the female chest and so breast growth secondary to hormone use appears smaller. Trans female hormone regimens induce erectile loss and oligo/ azoospermia. Trans women may also opt to have an orchidectomy and the surgical creation of a vagina. After transition of course, both trans men and trans women are best considered to be simply men or women, respectively, in circumstances where their trans status is irrelevant.

In both trans men and trans women therefore, loss of reproductive capability will accompany the use of cross-sex hormones. Although usually reversible if the person elects to stop taking the hormones, this loss may be irreversible. ${ }^{2}$ There will certainly be a permanent loss of reproductive function if the person has an orchidectomy or a hysterectomy and oophorectomy. Consequently, issues around the capacity for reproduction are highly important in the decision-making processes concerning trans people's transition, both for the individual and for the clinician. Several of the main bodies working in this field therefore recommend this topic for discussion between clinicians and people who are transitioning. ${ }^{23}$

The decision-making process around fertility for a trans person can be complicated by some people's perceptions around trans people having children at all. Trans people, and others, may feel that trans people are de facto not fit to have children, ${ }^{4}$ which may affect the decisions around transition for those trans people who already have children and who are considering living in another gender role; or the decision as to whether to store gametes prior to hormonal and/or surgical transition as in the examples above. As we shall see, parent(s) and caregivers being trans is in no way detrimental to children, 
but these matters do crop up in the clinic and so are discussed later.

\section{REPRODUCTIVE OPTIONS}

Within the Nottingham and Charing Cross gender clinics 'triadic therapy' is practised, meaning that more reversible changes are instigated before less reversible and irreversible ones. Thus one makes a change of gender role to present as one's felt gender and then starts hormones and then has chest surgery and then has genital surgery. Of course all of this is carefully managed, and an individual need not have all of these interventions if they do not want them. For the reasons given above, before initiating hormone or surgical treatment clients are counselled by psychologists, psychiatrists and/or endocrinologists regarding loss of fertility and they must consent to fertility loss before being recommended for hormone treatment.

Some trans men and women elect not to undergo genital surgery and/or removal of reproductive capacity and should they so choose they can therefore achieve pregnancy without assisted reproductive technologies. Hormonal interventions are often largely reversible, but it may take from 3 to 24 months after withdrawal of hormone treatment until reproductive functioning returns to normal. This means that those gendered characteristics that were being suppressed by a hormonal regimen will re-emerge and professional support is often crucial throughout this period. There may also be difficulties in accessing health care in such a gendered environment as reproductive medicine, and sensitive handling of this situation is important.

If trans people wish to store gametes, whether for use with a surrogate or with a fertile partner, harvesting is usually commissioned by the National Health Service (NHS) national commissioning for trans services, although cryostorage is usually undertaken privately as it is not financed by the NHS, and so has a cost implication that can be a barrier for some trans people. There is a reasonable chance of a live birth from trans women's sperm storage, in contrast to the chances for trans men who have approximately a $4.0-13.6 \%$ chance of a live birth from cryopreservation of their gametes, which is half the live birth rate from using fresh oocytes. ${ }^{5}$ Obtaining the gametes for cryopreservation is more invasive for trans men than for trans women, as standard oocyte induction and collection is needed, which can be both physically and psychologically arduous. Both trans men and trans women can feel dissuaded by the apparently gendered nature of the endeavour ${ }^{7}$ (trans women will often need to attend an andrology clinic, for example) and careful discussion around the implications of not undergoing that discomfort are most important. Similarly, some trans people misunderstand the nature of eggs and sperm, seeing them as inextricably gendered rather than merely vessels for strands of DNA. A more technical and less culturally construed consideration of these matters can help some trans people re-evaluate any culturally-received ideas they may have and so make a decision based on their reproductive wishes.

These discussions are extremely important for younger trans people who at the age of 18 years may make decisions, based on an understandable wish to transition as quickly as possible, which their 30 -year-old selves may regret when left without the possibility of having their own biologically-related children. Indeed, after starting hormones with the resultant attenuation of gender dysphoria, younger clients especially occasionally decide that they $d o$ wish to store gametes after all. Additionally, it is not uncommon for younger trans people to see adoption as a simple solution for their reproductive needs. ${ }^{4}$ While adoption is indeed a possibility for trans people, it is not without its difficulties due to the prejudices that still sadly persist in the minds of some professionals, and so should be thought through carefully with the individual. Many clients confuse the question of whether they wish to have the option of having children, which is the matter at hand, with whether they want to have children now, and this can usefully be disentangled in the consulting room.

Some trans people opt not to have gamete storage at any point as they have concerns that as they are trans they would not be fit parents This may be reflective of internalised transphobia, ${ }^{4}$ which is the state in which some trans people are themselves uncomfortable about, or phobic of, trans people. It is to this issue of trans parents that we now turn.

\section{CHILDREN}

There is no evidence that having a trans parent or caregiver harms children ${ }^{8-10}$ or indeed appears to affect their sexuality or gender. ${ }^{10}$ Although any acrimony between the parents or caregivers around the transition can lead to psychological difficulties for the children, this is not a trans-specific issue as acrimony between parents or caregivers on any matter can cause psychological difficulties for their children. ${ }^{8}$ Some professionals have argued that children of trans parents or caregivers may be subject to bullying and should therefore not be cared for by them. However, one would never think of removing a child from a minority ethnic or gay parent on grounds of bullying and the same should reasonably be held to be the case here. Instead, robust anti-bullying measures should be in place at the school. Indeed as trans identity is a protected characteristic in discrimination law, such discrimination would be construed as indirect transphobia. Arguments around it being parental choice to transition are false as, in most cases, the decision to transition is not based on choice but rather on a biological aetiology. ${ }^{4}$ Similarly false are notions that trans people have higher degrees of psychopathology than the general population. ${ }^{4}$ The literature and our own clinical experience shows that trans people are just as good 
parents as cisgender people (those who are content to remain the gender to which they were assigned at birth), and so have the same rights and responsibilities.

\section{CONCLUSIONS}

For those trans people who opt to make physiological changes involving hormones and surgery, infertility and reproductive decisions are an integral part of the transition and thus an integral part of the support that relevant professionals can offer to this client group. Trans people have the same rights and responsibilities as cisgender people and misunderstandings about the nature of trans people, by both trans people and professionals (who may, of course themselves be trans), should not prevent those trans people who wish to from storing gametes and having and caring for children.

Competing interests None.

Provenance and peer review Commissioned; externally peer reviewed.

\section{REFERENCES}

1 Seal LJ, Franklin S, Richards C, et al. Predictive markers for mammoplasty and a comparison of side effect profiles in transwomen taking various hormonal regimens. J Clin Endocrinol Metab 2012;97:4422-4428.

2 Hembree WC, Cohen-Kettenis P, Delemarre-van de Waal HA, et al. Endocrine treatment of transsexual persons: an Endocrine Society clinical practice guideline. J Clin Endocrinol Metab 2009;94:3132-3154.

3 Coleman E, Bockting W, Botzer M, et al. Standards of Care for the Health of Transsexual, Transgender, and Gender-Nonconforming People, Version 7. Inter J Trans 2012;13:165-232.

4 Richards C, Barker M. Sexuality and Gender for Mental Health Professionals: A Practical Guide. London, UK: Sage, 2013.

5 Borini A, Levi Setti PE, Anserini P, et al. Multicenter observational study on slow-cooling oocyte cryopreservation: clinical outcome. Fertil Steril 2010:1662-1668.

6 Medicine PCoASfR, Technology SfAR. Mature oocyte cryopreservation: a guideline. Fertil Steril 2013;99:37-43.

7 Wierckx K, Stuyver I, Weyers S, et al. Sperm freezing in transsexual women. Arch Sex Behav 2012;41: 1069-1071.

8 Freedman D, Tasker F, di Ceglie D. Children and adolescents with transsexual parents referred to a specialist gender identity development service: a brief report of key developmental features. Clin Child Psychol Psychiatry 2002;7:423-432.

9 White T. Adaptation and adjustment in children of transsexual parents. Eur Child Adolesc Psychiatry 2007;16:215-221.

10 Green R. Transsexuals' children. Inter J Trans 1998;2:97-103. 